\title{
EL TRABAJO EN ELAULA Y LA COMPETENCIA DIGITAL EN EL MODELO 1A1 DE LA COMUNIDAD DE MADRID
}

\section{WORK IN CLASSROOM AND DIGITAL COMPETENCE IN AN OLPC MODEL IN THE REGION OF MADRID}

\author{
Pablo Sánchez Antolín ${ }^{1}$ \\ pablo.sanchez@uclm.es \\ Tania Muñoz Álvarez² \\ tany_m_@hotmail.com \\ Dr. Joaquín Paredes Labra² \\ joaquin.paredes@uam.es
}

${ }^{1}$ Universidad de Castilla La Mancha. Facultad de Educación. Departamento de Pedagogía. Avenida de los Alfares, 42. 16071 Cuenca (España)

${ }^{2}$ Universidad Autónoma de Madrid. Facultad F. Profesorado y Educación. Departamento de Didáctica y Teoría de la Educación. Avda. Francisco Tomás y Valiente, 3, 28049

Madrid (España)

Se presenta un análisis del tipo de competencia digital incorporada por los estudiantes de primeros cursos de Educación Secundaria Obligatoria de la Comunidad de Madrid cuyos centros participan en un programa lal de introducción de un ordenador por estudiante. La metodología es un estudio intercasos. Hay observaciones de aula, entrevistas al profesorado observado, encuesta a estudiantes y análisis documental relevante. Se observan diferentes procesos de alfabetización para una competencia digital según la experiencia en proyectos con tecnologías de la información y la comunicación (TIC) de los profesores participantes. Asimismo, se observan pequeños espacios de resistencia y de cambio al modelo lal impulsado en Madrid.

Palabras clave: Usos de la tecnología en educación, métodos de enseñanza, alfabetización informática, competencia, Educación Secundaria.

This paper shows an analysis of digital competences obtained by students of first courses in Secondary Education of the Region of Madrid whose centers are participating in "one computer per student" program. The methodology used was study cases. It involved classroom observations, interviews with teachers, a survey to students and document analysis. Different processes for digital literacy competence are linked to experience of participating teachers in ICT projects. Also, small spaces of resistance and change into Madrid OLPC.

Keywords: Education Technologies, teaching methods, computer literacy, digital competences, secondary education.

Píxel-Bit. Revista de Medios y Educación. No 47 Julio 2015. ISSN: 1133-8482. e-ISSN: 2171-7966. doi: http://dx.doi.org/10.12795/pixelbit.2015.i47.14 


\section{Introducción y justificación del problema.}

La introducción de un ordenador por alumno, modelo OLPC (one laptop per child), 1:1 o 1a1 es una propuesta de «saturación tecnológica» (http://wiki.laptop.org/go/ Core_principles/lang-es) de los centros que tiene por objeto la experimentación de una enseñanza innovadora con tecnologías de la información y la comunicación (TIC). La saturación tecnológica es uno de los cinco principios clave de la existencia de un modelo OLPC, consistente en la presencia suficiente de TIC en los centros educativos, que cada estudiante disponga de un portátil.

Este dispositivo promueve una enseñanza democrática y pragmática, se ofrece a niños que cuidan de su equipo, lo llevan a todas partes y aprenden de forma ubicua. Junto al de «saturación», los conceptos fundamentales del modelo 1 a1 son los de «niño propietario», «niños de 6 a 12 años», «conectividad»y «abierto y libre».

Generalmente atiende los principios educativos de equidad social y alfabetización digital de la población. Es una realidad con predicamento internacional (http:// www.ceibal.org.uy). Es, quizá, la última frontera en la integración de las TIC en las escuelas que, desafortunadamente, la situación económica ha truncado. Tiene presencia española mediante diversos programas regionales (Losada, Karrera \& Correa, 2011). Se trata de innovaciones a gran escala, fruto de una política de integración de las TIC, y con un plan de dotación, formación, apoyo, materiales y modificaciones curriculares.

El problema de la competencia digital ha sido objeto de un largo debate en los últimos 15 años (Esteve, Duch \& Gisbert, 2014), promovido por las instituciones internacionales, los académicos y, finalmente, los gobiernos interesados en concretar mediante políticas el tipo de integración de las TIC que se habría de producir en las escuelas y el tipo de preparación de sus docentes para afrontarla con sus estudiantes.

Algunos gobiernos regionales españoles, como el de la Comunidad de Madrid, han hecho concreciones de estas políticas -en este caso ciertamente radicales, pues han rechazado la política nacional y han generado su propia política, discurso y decisionesEstas concreciones en el denominado «Programa de institutos de innovación tecnológica» han sido el tipo de dotaciones (donde se optó por terminales en vez de laptops, quizá lo más llamativo del programa madrileño) y el tamaño de la innovación (experimental, menos del 5\% de los centros públicos que imparten $1^{\circ}$ y $2^{\circ}$ de la Educación Secundaria Obligatoria, ESO, cuando en el resto de regiones fue generalizado para esos dos cursos, o en su caso a $5^{\circ}$ y $6^{\circ}$ de Educación Primaria).La diferenciación de los centros a través de programas de innovación que incorporan mayores dotaciones, como se da en estos institutos, responde a una política de la Comunidad de Madrid que busca una mayor competitividad entre éstos, bajo la falsa premisa de que se producirá un aumento de la calidad educativa (Prieto \& Villamor, 2012).

Las políticas madrileñas sobre integración de las TIC abogan por que la competencia digital supone maestría en el dominio de las TIC por parte de los estudiantes, una forma de ejercitar la competencia digital que se ha de traducir en una mejora en el rendimiento instructivo (Paredes, 2012). Pero, ¿realmente es éste el resultado de la competencia digital que plantea la investigación reciente y que recoge la literatura sobre el tema? 
Hubo un movimiento para fijar competencias digitales de los niños y jóvenes (entre otras iniciativas, la licencia europea para utilizar ordenadores Epict y otra de una conocida empresa de software), y en 2006 se definió para toda España esta competencia por vez primera -pues hubo un propuesta anterior para Cataluña denominada Actic(Real Decreto 1513/2006, de 7 de diciembre, por el que se establecen las enseñanzas mínimas de la Educación Primaria).

La literatura reciente en español parece que tiene una perspectiva más amplia de esta competencia. Alonso (2011) plantea que su concreción en el currículo es limitada, pues sólo contempla habilidades, actitudes y conocimientos de cara a la información, dejando de lado uno de los principios básicos de la Web 2.0, la producción de conocimiento. Del Moral y Villalustre (2011), al analizar la competencia digital entre maestros rurales, constatan la transformación de la competencia digital para los jóvenes y en los últimos años a cuenta de un nuevo papel para una tecnología social integrada en la comunidad. González-Martínez, Espuny, De Cid y Gisbert (2012), al construir una herramienta para la evaluación de la competencia digital en Educación Secundaria, ofrecen la perspectiva ampliada de Larraz sobre la competencia digital, que supone: tratamiento de datos en diferentes formatos, gestión de la información (reconocer, localizar, evaluar, organizar, transformar, comunicar), comunicación (participación activa, civismo, identidad digital), análisis de mensajes multimedia (acceso, comprensión y análisis crítico), elementos todos ellos imbricados con el resto. Algo más allá de un uso instrumental. Hace falta un sentido más pleno, actitudinal y social, y menos instrumental de lo que supone la competencia digital, que se aproxima a haber recibido una educación en medios (Gutiérrez \& Tyner, 2012). La masiva presencia de los medios y dispositivos en la vida cotidiana debería tener un correlato, una aplicación y un análisis en los espacios escolares.

Además de ampliar la mirada a lo que es la competencia, y como ya se planteó en la polémica Clark-Kozma (Schmid et al., 2009), frente a los análisis o evaluaciones de corte técnico, conviene analizar si es posible ir más allá del recuento y valoración de los usos. Convendría acercarse a lo que los sostiene y dificulta, un estudio evaluativo y de casos (Becta, 2004; Valverde, Garrido \& Sosa, 2010; Sancho \& Alonso, 2011) necesario para establecer otros análisis iluminativos de qué ocurre en la implantación de un programa como el 1a1 en la Comunidad de Madrid.

Esta investigación forma parte de una fase de un estudio más amplio, financiado por el Ministerio de Ciencia e Innovación (Ref. EDU2010-17037), titulado «Las políticas de un «ordenador por niño» en España. Visiones y practicas del profesorado ante el programa escuela 2.0". Aquí se presentan resultados de la segunda fase del estudio, donde los autores realizan un examen en profundidad de la puesta en marcha del modelo 1a1 en la región de Madrid y de la vida en sus aulas. Los objetivos de la segunda fase del estudio macro fueron:

Analizar los cambios en los aspectos organizativos de los procesos de enseñanza provocados por la presencia masiva de las TIC tanto en el centro escolar como las aulas ordinarias.

Identificar qué tipo de actividades didácticas son realizadas con las TIC (pizarras digitales, Web 2.0, ordenadores personales) y demás materiales didácticos (libros de texto, especialmente) en las aulas. 
Explorar los efectos de las TIC sobre el desarrollo de competencias digitales e informacionales en el alumnado.

Valorar en qué medida la presencia masiva de las TIC está innovando el modelo y prácticas de enseñanza-aprendizaje desarrolladas por el profesorado.

El objetivo específico para este subestudio que presentamos fue comprender el tipo de actividades que hacen los profesores para que los estudiantes trabajen la competencia digital, con las peculiaridades de la propuesta regional a las que se aludió al principio, y cómo lo hacen en la región de Madrid.

\section{Metodología.}

La metodología para este subestudio es de tipo cualitativo, un estudio intercasos elaborado a partir de los estudios de caso desarrollados por los distintos miembros del equipo de investigación en la región de Madrid (CAM). Dichos informes suponen un estudio de casos de generalmente uno, aunque en varios de ellos dos profesores por centro que imparten su docencia en los cursos de $1^{\circ}$ y $2^{\circ}$ de la ESO (excepcionalmente en $5^{\circ}$ y $6^{\circ}$ de Primaria, por la naturaleza específica de este tipo de programas en Madrid, que decidió aplicarlo en ESO, en vez de en Primaria). Son profesores que participan en el programa que promueve la región de Madrid, con experiencia en la introducción de TIC en la educación, acreditada en un concurso entre los más de 200 centros que concurrieron a la ayuda, y con la que se premió a 15.

La metodología supone la realización de observaciones de aula, entrevistas al profesorado observado, entrevistas a los coordinadores TIC, encuesta a estudiantes y análisis documental relevante.
La unidad de análisis son 7 centros de Educación Secundaria Obligatoria, seis públicos y uno privado. Participan voluntariamente en esta fase del estudio. En cada centro se ha procurado que participaran al menos dos docentes participantes en el «Programa de institutos de innovación tecnológica», con un docente «experto» (que fuese su segundo año) y otro «novel» (primer año en el citado programa). Habida cuenta de que este plan cuenta con 15 centros públicos, se trata de un muestreo muy amplio. Cada caso se estudió a lo largo del curso 2011-12.

El proceso de acceso fue negociado con cada uno de los equipos directivos de los centros participantes. La observación realizada en las aulas fue diseñada a nivel nacional por el equipo de 40 investigadores participantes, fue no sistemática, recogiendo información de la actividad de docentes y estudiantes en las mismas. Los observadores acudieron durante varios meses a las aulas y tomaron nota de las secuencias de actividades, materiales utilizados e interacciones en las aulas.

Las entrevistas fueron generadas a partir de un protocolo diseñado desde una revisión de literatura y validado por jueces, sobre cuatro dimensiones (organizativas, didácticas, de aprendizaje y de desarrollo profesional del docente) del trabajo de los docentes en las aulas.

A los coordinadores les fue requerida cierta información sobre el origen de la propuesta realizada por cada centro, las actividades previas, la formación de los docentes antes y durante la puesta en marcha del proyecto, las ventajas y dificultades y otros aspectos que permitieran conocer el contexto de aplicación de los programas. Estas dimensiones también fueron discutidas a nivel nacional por el resto 
de equipos y para sus respectivos programas regionales.

El cuestionario de estudiantes recogió información sobre sus preferencias en relación con un elenco de actividades que ejemplifican la competencia digital, a partir de un instrumento nacional más amplio generado para el estudio como un nuevo subestudio. En el cuestionario aplicado en Madrid se utilizó una pregunta que enumera las posibles actividades con TIC en el aula. Fue aplicado en tan sólo 3 de los 7 centros-caso, por dificultades en alguno de los centros para continuar recogiendo información.

Se consultaron las memorias de centro y los proyectos con los que concurrieron los equipos que promueven cada innovación.

También se realizaron fotografías de las aulas vacías, recogiendo la disposición de los equipos y otros aspectos que incluye el entorno de aprendizaje que constituyen.

Para el análisis de toda esta información se siguió un proceso de transcripción de las entrevistas y observaciones, así como de vaciado documental, se elaboraron listados y matrices de elementos con ayuda de cuatro dimensiones definidas previamente por el equipo investigador nacional y se inició un proceso de diálogo y comparación constante con los resultados de cara a interpretarlos y construir un relato explicativo triangulado y complejo (Stake, 2007). Los datos de la encuesta fueron tabulados y graficados para su interpretación. Las fotografías fueron valoradas y formaron parte de informes parciales.

Se construyeron informes que se compartieron con los docentes participantes en el estudio, que los comentaron.

\section{Resultados.}

En Madrid, y para unos pocos centros de Educación Secundaria, se ha hecho una dotación masiva de TIC (Figura 1). Se ha previsto que la presencia de esa TIC apoye

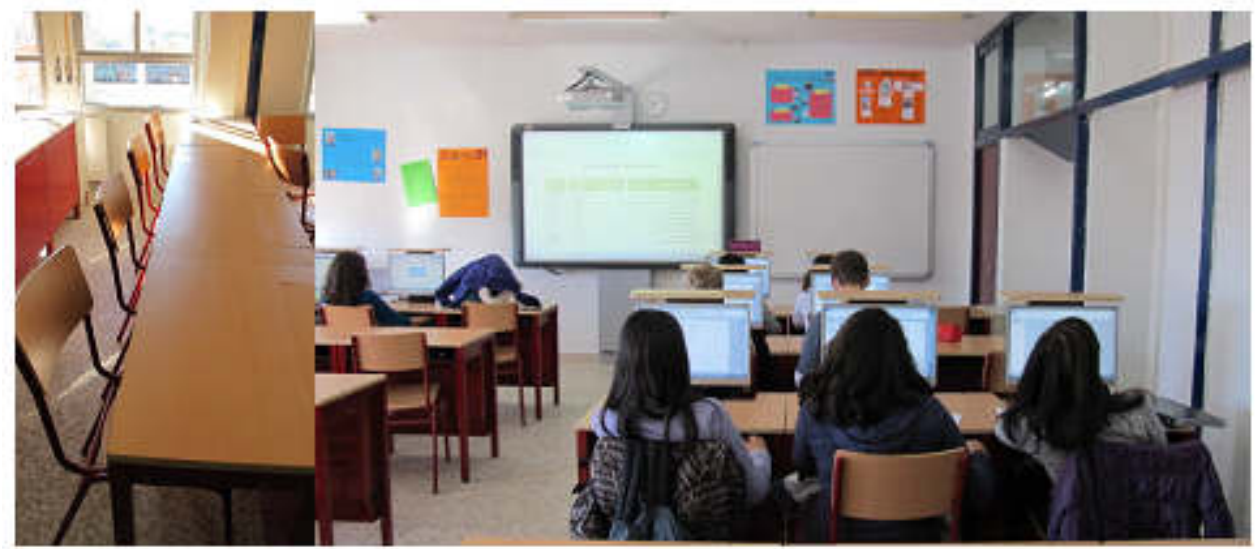

Figura 1. Un aula del programa de institutos tecnológicos de Madrid vista en dos momentos: antes (como aula regular, con monitores y teclados guardados en cada pupitre) y cuando se pone a trabajar con ordenadores (con los monitores emergidos y los teclados extraídos del pupitre). Fuente: archivo fotográfico de los investigadores.

Píxel-Bit. Revista de Medios y Educación. No 47 Julio 2015. ISSN: 1133-8482. e-ISSN: 2171-7966. doi: http://dx.doi.org/10.12795/pixelbit.2015.i47.14 
que en las materias que cursan los estudiantes haya suficiente tiempo para analizar contenidos con herramientas TIC. Está previsto para al menos un $30 \%$ del horario semanal de varias materias entre las que obligatoriamente tienen que estar Matemáticas y Tecnología.

Para entender la forma de trabajar la competencia TIC en los centros de Secundaria de la región de Madrid hay que comprender la forma en que se trabaja en las clases.

El trabajo de los estudiantes y, en general, la naturaleza de su interacción con las TIC con objeto de trabajar la competencia digital está supeditado a la lógica de la dirección de la enseñanza, la naturaleza de la relación pedagógica (Hernández, 2011), la posición epistemológica del docente y el empleo de espacio y tiempo.

Las TIC no son determinantes para el tipo de interacción en el aula. Se observa que cuando no disponen de ordenadores (en una parte del horario) son profesores con la misma vocación ilustrativa que cuando los tienen, que les gusta mantener un nivel de interacción muy alto con todos los estudiantes, que están pendientes de incluirles en la clase.

Cuando disponen de ordenadores, pueden dirigir totalmente la actividad de la clase con los mismos.

Esta dirección es posible por el dispositivo técnico incorporado en las aulas. Para ello, el puesto del profesor está equipado con un ordenador conectado a dos pantallas, una de ellas táctil que le permite interactuar con los equipos de los alumnos. De esta forma, tiene el control de los equipos y puede supervisar en cualquier momento el trabajo de los alumnos en tiempo real (entrevista PPFLP, 1 diciembre 2011).
La forma en que los docentes trabajan hace ver que la dirección de la enseñanza les corresponde fundamentalmente a ellos, y los estudiantes les siguen:

El profesor da instrucciones verbales para que localicen la actividad: Prof.: Vais abriendo el ordenador y entráis en el aula virtual (...) Muestra en la pizarra digital dónde está la actividad (...) Presenta la tarea antes de que se lleve a cabo (...) Realiza aclaraciones conforme se van produciendo dificultades en la ejecución de la tarea (...) Refuerza positivamente logros y descubrimientos (habitualmente, PPFLPJL, 26 marzo 2012).

Los estudiantes nunca pueden emprender actividades por su cuenta en el aula. Hay un plan de trabajo marcado por el programa y el libro de texto. Hay incluso rutinas como salir a resolver problemas a la pizarra digital, por un orden de la clase.

Los estudiantes están sometidos a la lógica del programa. Sólo pueden abrir el ordenador cuando el profesor lo autoriza, tiene un fin productivo, orientado a una tarea.

Con respecto a la posición epistemológica del docente, ésta también condiciona las posibilidades de exploración y experimentación con los dispositivos:

Mis alumnos deben saber matemáticas con regla y compás y también con el programa Geogebra (un software de matemáticas) (entrevista PPFLPAL, 16 diciembre 2011).

Otros handicaps en la actividad de los estudiantes son el espacio y el tiempo. En el modelo 1a1 de la región de Madrid los ordenadores están empotrados en los pupitres, y estos están anclados al suelo. La única forma de trabajar es frente a las pizarras velleda y digital, individualmente o por parejas. Esta disposición física es ideal para limpiar las aulas y mantener un control tradicional de la misma, pero es poco útil para 
cualquier metodología no magistral o que esté orientada a desplegar todos los elementos de una alfabetización para una competencia digital amplia.

Los procesos de exploración libre no son bien recibidos. Los profesores manifiestan cierto miedo a la actividad más elaborada a que daría pie un uso indiscriminado de TIC:

Los profesores, cuando se meten en las aulas, lo que tienen es miedo a los alumnos. Les pide a sus profesores que lo inviertan, que aprovechen la sabiduría de los estudiantes (entrevista PPFLP, 1 diciembre 2011).

El alumnado trabaja sólo en los momentos que se le marcan, haciendo uso de las TIC. Hay un alumno en cada puesto. Cuando necesitan asesoramiento lo piden y resuelven las dudas que tengan sobre una tarea concreta propuesta por el profesor.

Sus actividades con TIC, recogidas a través la observación son: Pasar a limpio algo hecho manualmente. Buscar información necesaria. Discernir fuentes de información. Utilizar un audio como fuente de información. Planificar el desarrollo de la tarea. Leer para toda la clase. Resolver un problema en un simulador. Experimentar (para luego resolver). Compartir hallazgos. Hacer ejercicios. Copiar un ejercicio para realizarlo en el cuaderno. Resolver cuestionarios de tipo autoevaluativo $\mathrm{y}$ formativo.

Los productos TIC elaborados por los estudiantes en clase han sido variados, aunque generalmente no pertenecen al desarrollo de proyectos o a la indagación. Son cuadernos de apuntes, hojas de respuestas a preguntas, ejercicios, tablas, informes, redacciones, presentaciones, operaciones matemáticas, pruebas objetivas en el aula virtual, trabajos elaborados en la PDI. También hay algunas actividades de investigación: webquest, blogs, wikis, exploración o búsqueda pautada, trabajo monográfico, investigación utilizando Internet. Estas últimas han contribuido a desarrollar la competencia de autonomía personal y la responsabilidad en sus propias tareas.

Las actividades observadas en las aulas están relacionadas con una parte de la competencia digital (Larrazen GonzálezMartínez et. al., 2012), el manejo de la información.

Se observan algunas diferencias entre los profesores en función de su experiencia en este programa que tienen repercusiones en los aspectos de competencia digital implicados en sus actividades. Así, los profesores «novatos» se tienen que enfrentar con el desafío de armar un currículo con actividades específicas, mientras van dominando las herramientas de la clase y comprendiendo la dinámica del proyecto. Estas actividades suelen ser de ejercitación y búsqueda de información. Para algunos profesores «expertos», como es el caso de los coordinadores proponentes de los proyectos, gente muy formada y de largo recorrido en la introducción de las TIC, el catálogo de procedimientos y técnicas empleado en las clases es rico:

Buscar, investigar, pensar, resolver situaciones, respeto, compartir, ayudar, integrar a otros niños, relajar a los adultos en sus miedos (entrevista PPFLPJL, 1 diciembre 2011).

Los profesores expertos conducen, además, un trabajo de alfabetización mediática (Gutiérrez y Tyner, 2012):

Son alumnos pequeños, igual no abren lo que deben... (hay que) educarles en la responsabilidad.... (entrevista PPFLPAL, 16 diciembre 2011). 
Esto nos hace sospechar que no estamos asistiendo al mero rearme de modelos transmisores ni a un trabajo educativo para una competencia digital exclusivamente instrumental o de contenido informativo.

Una profesora, al leer el informe etnográfico, nos confiesa cambios experimentados en su rol:

Lo que me ha hecho replantearme es que antes yo era muy cuadriculada y ponía exámenes, ahora hago más trabajos, pero me doy cuenta de que no tienen que saber más por hacer más exámenes, del examen final no se libran... con los pequeños $\left(1^{\circ} \mathrm{ESO}\right)$ hemos estado haciendo un cuaderno con las medidas, vi en internet un cuaderno digital, cada cuaderno tenía como 3 fichas, los puestos individuales cada uno trabaja más. Ahora es cuando les doy más tiempo para trabajo en clase. De antes a ahora ha abierto un tiempo para hacer ejercicios en clase. 12 cuadernos de sistema métrico, todos los han hecho, en clase 8 cuadernos y 4 en casa. Otros años hacía unas fichas en clase, pero no funcionaban igual, quedaban muchas en blanco (entrevista PPFLPAL, 18 abril 2012).

Relajar ritmos (a pesar de seguir obsesionada por los resultados, por la propia

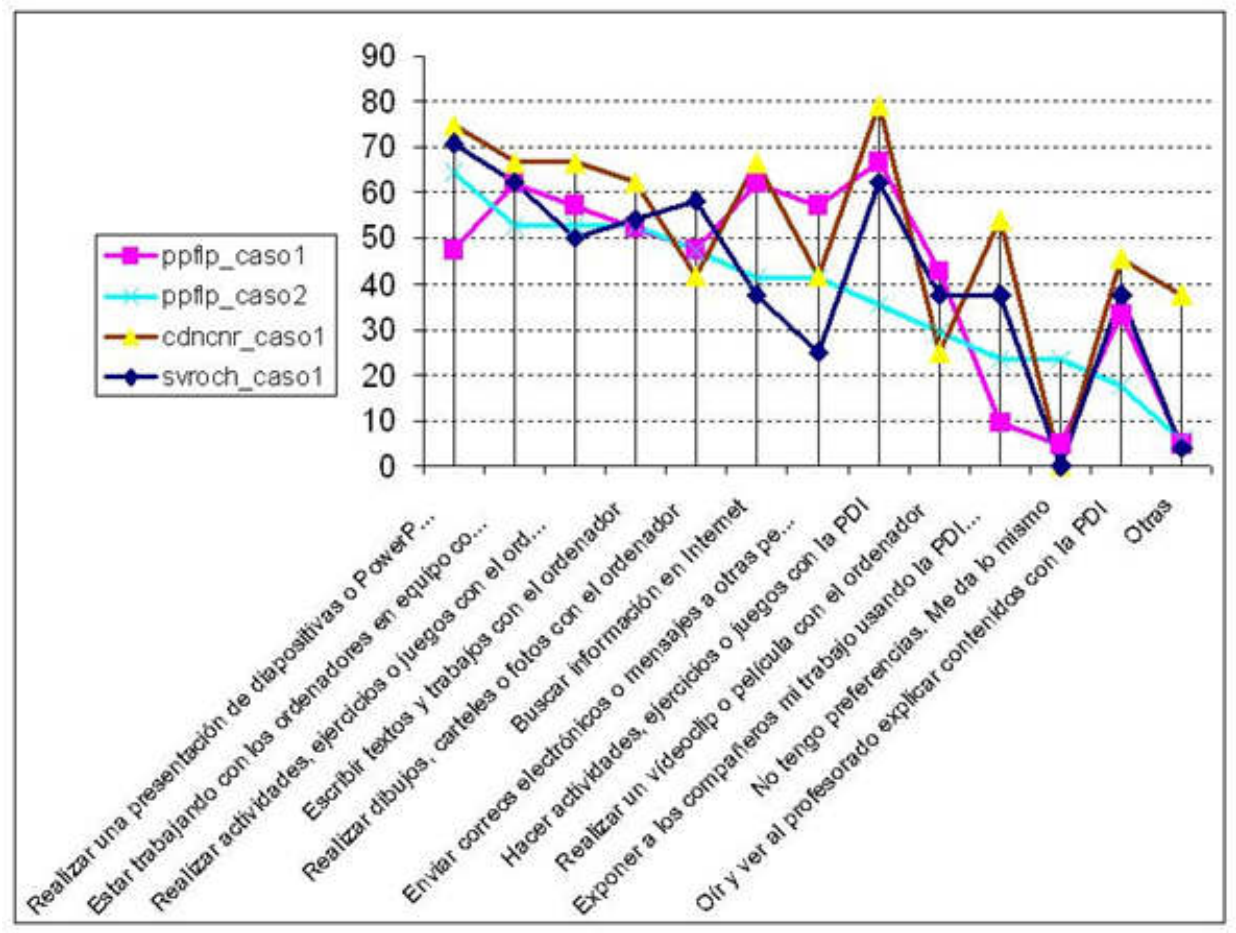

Gráfico 1. Valoración de las preferencias de actividades con TIC de los estudiantes de los casos analizados en el estudio sobre el modelo lal en la Comunidad de Madrid. Porcentaje de estudiantes que indican esa preferencia Fuente: elaboración propia a partir de los datos de una encuesta aplicada en las aulas participantes. 


\begin{tabular}{|c|c|c|c|c|}
\hline $\begin{array}{l}\text { Realizar una presentación de diapositivas o } \\
\text { PowerPoint }\end{array}$ & $\begin{array}{c}\text { svroch_casol } \\
70,8\end{array}$ & $\begin{array}{c}\text { ppflp_caso1 } \\
47,6\end{array}$ & $\begin{array}{c}\text { cdncnr_caso1 } \\
75,0\end{array}$ & $\begin{array}{c}\text { ppflp_caso2 } \\
64,7\end{array}$ \\
\hline $\begin{array}{l}\text { Estar trabajando con los ordenadores en equipo } \\
\text { con un grupo de compañeros } \\
\text { Realizar actividades, ejercicios o juegos con el } \\
\text { ordenador personal }\end{array}$ & $\begin{array}{l}62,5 \\
50,0 \\
\end{array}$ & $\begin{array}{r}61,9 \\
57,1 \\
\end{array}$ & $\begin{array}{l}66,7 \\
66,7 \\
\end{array}$ & $\begin{array}{l}52,9 \\
52,9 \\
\end{array}$ \\
\hline Escribir textos y trabajos con el ordenador & 54,2 & 52,4 & 62,5 & 52,9 \\
\hline Realizar dibujos, carteles o fotos con el ordenador & 58,3 & 47,6 & 41,7 & 47,1 \\
\hline $\begin{array}{l}\text { Buscar información en Internet } \\
\text { Enviar correos electrónicos o mensajes a otras } \\
\text { personas por Internet }\end{array}$ & $\begin{array}{l}37,5 \\
25,0 \\
\end{array}$ & $\begin{array}{r}61,9 \\
57,1 \\
\end{array}$ & $\begin{array}{l}66,7 \\
41,7 \\
\end{array}$ & $\begin{array}{l}41,2 \\
41,2 \\
\end{array}$ \\
\hline Hacer actividades, ejercicios o juegos con la PDI & 62,5 & 66,7 & 79,2 & 35,3 \\
\hline $\begin{array}{l}\text { Realizar un vídeoclip o película con el ordenador } \\
\text { Exponer a los compañeros mi trabajo usando la } \\
\text { PDI en clase }\end{array}$ & $\begin{array}{l}37,5 \\
37,5\end{array}$ & $\begin{array}{l}42,9 \\
9,5 \\
\end{array}$ & $\begin{array}{l}25,0 \\
54,2 \\
\end{array}$ & $\begin{array}{l}29,4 \\
23,5\end{array}$ \\
\hline $\begin{array}{l}\text { No tengo preferencias. Me da lo mismo } \\
\text { Oír y ver al profesorado explicar contenidos con } \\
\text { la PDI }\end{array}$ & $\begin{array}{r}0,0 \\
37,5 \\
\end{array}$ & 33,3 & 45,8 & $\begin{array}{l}23,5 \\
17,6 \\
\end{array}$ \\
\hline Otras & 4,2 & 4,8 & 37,5 & 5,9 \\
\hline
\end{tabular}

Tabla 1. Valoración de las preferencias de actividades con TIC de los estudiantes de los casos analizados en el estudio sobre el modelo lal en la Comunidad de Madrid. Porcentaje de estudiantes que indican esa preferencia Fuente: elaboración propia a partir de los datos de una encuesta aplicada en las aulas participantes.

presión de la administración educativa), permitir la actividad en el aula, pasar de un modelo de mera transmisión a otro de ejercitación, no son grandes cambios, pero suponen un nuevo espacio de trabajo.

Aparecen (a tenor de lo declarado por los profesores) otros comportamientos docentes, otra cultura de centro, el profesorado se coordina y hace actividades en común.

Otros aspectos que señala el profesorado sobre logros y competencia digital:

Yo creo que el que aprende, aprende mucho mejor, y no se olvida (STMBJM, marzo 2012).

Los resultados no son objetivos... son resultados que el profesor percibe en la motivación, en el trabajo de determinadas destrezas. Yo siento que los alumnos están aprovechando eso para ir más rápido, y más cómodos y más seguros... entonces me parece muy positivo (GGR, febrero 2012).

Favorece la regulación del ritmo de aprendizaje de los alumnos, al igual que desarrolla la competitividad y la ejercitación de habilidades y destrezas (GGR, marzo 2012).

Por otra parte, una encuesta realizada en 3 (uno con dos aulas) de los 7 centros participantes en el subestudio de Madrid nos permite valorar la percepción de la actividad en el aula por parte de los estudiantes (Gráfico 1 y Tabla 1) y el tipo de actividades orientadas a trabajar su competencia digital.

Las actividades que más gustaron, de las que se ofrecieron para su valoración, fueron hacer actividades, ejercicios o juegos con la pizarra digital interactiva, estar trabajando con los ordenadores en equipo con un grupo de 
compañeros y realizar una presentación de PowerPoint.

Se trata de estudiantes que, en un contexto de enseñanza dirigida, con un programa rígido y sin preparación para un trabajo autónomo, prefieren software con el que se manejan de manera intuitiva (aunque sea escolar, como el de la PDI) y actividades de exploración, a actividades con TIC más tradicionales. Les gustan actividades más exploratorias y de construcción.

\section{Discusión.}

El objetivo específico para este subestudio que presentamos fue comprender el tipo de actividades que hacen los profesores de Madrid para que los estudiantes de la ESO trabajen la competencia digital, con las peculiaridades de la propuesta regional.

El estudio ofrece en primer lugar una comprensión del sentido de la actividad docente en las aulas.

No hay una transformación de la vida en las aulas ni se renueva el papel de los estudiantes. Los productos generados por los estudiantes son comprobaciones de aprendizajes, no investigaciones o proyectos. La apuesta por la calidad y la necesidad de evaluar de forma sistemática los aprendizajes de los estudiantes no favorece inicialmente otros objetivos, pongamos por caso transversales, que pudieran exigir ir más allá de lo meramente transmisivo y, entonces, reorganizar la vida en las aulas. Es un condicionante importante para conseguir una competencia con TIC en los términos tan amplios arriba descritos.

Aparecen ciertos espacios de apertura a otra enseñanza (y otros contenidos de la competencia digital) dentro de un contexto de pervivencia de un modelo directivo, que hacen plantearse que quizá se pueda avanzar hacia el abordaje en las aulas de Secundaria de otros componentes de la competencia digital, ligados a la alfabetización mediática.

Los docentes se resisten a la obsesión por los resultados. Parece que emerge una propuesta renovada de relación con el conocimiento, donde los estudiantes pueden tener un papel nuevo, y su competencia digital una función bien específica de apropiación y reconstrucción del conocimiento, apoyada en la retroalimentación que reciben de los estudiantes: motivación, confianza, autonomía, dominio de determinadas destrezas.

Con respecto a los resultados, una parte del profesorado identifica progresos notables en algunos elementos de la competencia digital. Sin embargo, no perciben que se esté provocando un incremento del rendimiento del alumnado. Algún profesor está entrenando a los estudiantes con exámenes ad hoc para poder enfrentar mejor los controles administrativos.

El profesorado aborda la competencia digital dentro de un modelo innovador nuevo, a caballo entre lo deseado y lo posible (Montero \& Gewerc, 2010), que resquebraja otro impuesto, que sólo pretende mejorar los resultados académicos, y más tradicional. Este cambio de modelo puede acabar influyendo en la profundización de los grados de dominio de la competencia digital que se trabaja en las aulas de la región de Madrid.

En relación con otros programas 1a1 en España, la actividad en las aulas (y fuera de ellas) y el trabajo de los docentes están condicionados por la forma de interpretar el modelo 1a1 en la región de Madrid.

Al buscar la diferenciación de las políticas de dotación con otras regiones, los principios clave del modelo 1a1, según los concibe la 
comunidad internacional, se han modificado $\mathrm{y}$, con ellos, tanto el tipo de actividad de los estudiantes como los resultados posibles. Frente a una alfabetización inicial y globalizada, su utilización en Primaria, la búsqueda de niños autónomos y la ubicuidad, hay usos especializados con un software propietario, participan niños de Secundaria, la responsabilidad es del centro, no hay un plan regional de conectividad de las escuelas y hogares y se organiza en terminales en red anclados al suelo de las aulas. Se le ha dado la vuelta a los principios de un programa OLPC, salvo la idea de saturación.

Ahora bien, a pesar del sesgo de las políticas puestas en práctica en Madrid y a falta de la compleción de otros sub-estudios similares a éste en otras regiones, los resultados de la actividad que declaran los profesores a través de un cuestionario mostró, en general y para el país, la prevalencia de la exposición de contenidos y la ejercitación de los estudiantes. Se está, entonces, muy lejos de una actividad creativa y colaborativa posibilitada por los dispositivos de que disponen, con una perspectiva más amplia y orientada al trabajo. Sólo así sería posible favorecer la adquisición de la competencia digital buscada.

\section{Fuentes de financiación.}

Esta investigación forma parte de una fase de un estudio más amplio, financiado por el Ministerio de Ciencia e Innovación (Ref. EDU2010-17037), titulado «Las políticas de un «ordenador por niño» en España. Visiones y prácticas del profesorado ante el programa escuela 2.0".

\section{Referencias bibliográficas.}

Alonso, A. (2011). El desarrollo del concepto de competencia digital en el currículum de las enseñanzas obligatorias de Galicia. Innovación educativa, 21, 151-159.

Becta (2004). Getting the most from your interactive whiteboard: A guide for secondary schools. Coventry, Reino Unido: British Educational Communications and Technology Agency.

Del Moral, E. \&Villalustre, L. (2011). Digitalización de las escuelas rurales asturianas: maestros rurales 2.0 y desarrollo local. Profesorado: Revista de curriculum y formación del profesorado, 15 (2), 109-123.

Esteve, F.M.,Duch, J. \& Gisbert, M. (2014). Los aprendices digitales en la literatura científica: diseño y aplicación de una revisión sistemática entre 2001 y 2010. Píxel-Bit. Revista de Medios y Educación, 45, 9-21.doi: http://dx.doi.org/10.12795/pixelbit.2014.i45.01

González-Martínez, J.,Espuny, C., De Cid, M$^{\mathrm{a}}$.J.\&Gisbert, M. (2012). INCOTIC-ESO. Cómo autoevaluar y diagnosticar la competencia digital en la Escuela 2.0. Revista de investigación educativa, 30 (2), 287-302.

Gutiérrez, A.\&Tyner, K. (2012). Educación para los medios, alfabetización mediática y competencia digital. Comunicar: Revista científica iberoamericana de comunicación y educación, 19 (38), 31-39. doi: http:// dx.doi.org/10.3916/C38-2012-02-03

Hernández, F. (Coord.) (2011). Pensar la relación pedagógica en la universidad desde el encuentro entre sujetos, deseos y saberes. Barcelona: Indaga-t-Recerca. Recuperado de http://fint.doe.d5.ub.es/ indagat-web/

Losada, D., Karrera, I. \& Correa, J. M. (2011). Políticas sobre la integración de las TIC en la escuela de la Comunidad Autónoma 
del País Vasco. Relatec: Revista Latinoamericana de Tecnología Educativa, 10 (1), 21-35.

Montero, L.\& Gewerc, A. (2010). De la innovación deseada a la innovación posible. Escuelas alteradas por las TIC. Profesorado: Revista de curriculum y formación del profesorado, 14 (1), 303-318.

Paredes, J. (2012). Políticas educativas neoliberales para la integración de las TIC en educación. El caso de Madrid (España). Campus Virtuales. Revista científica iberoamericana de tecnología educativa, 1 (1), 11-20.

Prieto, M. \&Villamor, P. (2012). Libertad de elección, competencia y calidad: las políticas educativas de la comunidad de Madrid. Profesorado. Revista de currículum y formación del profesorado, 16 (3), 127-144.

Real Decreto 1513/2006, de 7 de diciembre, por el que se establecen las enseñanzas mínimas de la Educación primaria(BOE núm. 293, 7 de diciembre de 2006).
Sancho, J.\& Alonso, C. (Coords.) (2011). Cuatro casos, cuatro historias de uso educativo de las TIC. Barcelona: Universitat de Barcelona. Recuperado de http:// hdl.handle.net/2445/17122

Schmid, R. (2009). Technology's effect on achievement in higher education: a Stage I meta-analysis of classroom applications. Journal of Computing in Higher Education, 21, 95-109.doi: 10.1007 / s12528-009-9021-8

Stake, R. (2007). Investigación con estudio de casos. Madrid: Morata.

Valverde, J., Garrido, C. \& Sosa, M. J. (2010). Políticas educativas para la integración de las TIC en Extremadura y sus efectos sobre la innovación didáctica y el proceso enseñanzaaprendizaje: La percepción del profesorado. Revista de Educación, 352, 99-124.

Fecha de recepción: 17-02-2015

Fecha de evaluación: 12-03-2015

Fecha de aceptación: 17-03-2015 